\section{Funcionamento familiar e dependência da internet em adolescentes}

\section{Family functioning and internet addiction in adolescents}

\section{Funcionamiento familiar y adicción a internet en adolescentes}

\author{
Daisy Araújo Rodrigues ${ }^{1 凶} \underline{\mathrm{ORCID}}$; Inês Carvalho Relva ${ }^{2 凶} \underline{\mathrm{ORCID}}$; Otília Monteiro Fernandes ${ }^{3 凶} \underline{\mathrm{ORCID}}$ \\ ${ }^{1}$ Mestre em Psicologia da Educação e do Desenvolvimento pela Universidade de Trás-os-Montes e Alto Douro, Portugal. \\ 2 Doutorada em Psicologia. Professora no Departamento de Educação e Psicologia na Universidade de Trás-os-Montes e Alto Douro, \\ Portugal; Membro do Centro de Investigação em Desporto, Saúde e Desenvolvimento Humano. Fundos nacionais apoiam o trabalho \\ da autora Inês Relva através da FCT - Fundação Portuguesa para a Ciência e Tecnologia no âmbito do projeto Estímulo ao Emprego \\ Científico - Chamada Institucional - CEECINST /00127/2018. \\ ${ }^{3}$ Doutorada em Psicologia. Professora no Departamento de Educação e Psicologia na Universidade de Trás-os-Montes e Alto Douro; \\ Membro Integrado do Centro de Investigação em Psicologia para o Desenvolvimento (CIPD).
}

Fecha correspondencia:

Recibido: octubre 08 de 2020.

Aceptado: julio 30 de 2021.

Forma de citar:

Rodrigues, D. A., Relva, I. C., \&

Fernandes, O. M. (2022).

Funcionamento familiar e dependência da internet em adolescentes. Rev. CES Psico, 15(1), 44-67. https://dx.doi.org/ $\underline{10.21615 / \text { cesp. } 5900}$

\section{Open access}

C) Derecho de autor

Licencia creative commons

Ética de publicaciones

Revisión por pares

Gestión por Open Journal System

DOI: $10.21615 /$ cesp.5900

ISSNe: 2011-3080

Publica con nosotros

\section{Resumo}

O presente estudo teve como objetivo principal explorar de que forma variáveis do funcionamento familiar se relacionam com a dependência da internet. A amostra final foi constituída por 568 participantes, com idades compreendidas entre os 14 e 18 anos, que frequentam o Ensino Secundário, num distrito da região Norte de Portugal. Foi usado um Questionário Sociodemográfico, o Internet Addiction Test (IAT) e o Systemic Clinical Outcome Routine Evaluation (SCORE-15). Verificou-se que $73.1 \%$ dos participantes apresentaram dependência leve a moderada, mas não foram encontradas diferenças na dependência da internet entre o sexo masculino e o feminino. Por outro lado, verificaram-se diferenças a nível de funcionamento familiar, sendo que o sexo masculino apresentou, ligeiramente, mais dificuldades na comunicação na família e dificuldades familiares. Observaram-se diferenças entre os grupos em estudo (utilizadores normais, utilizadores com dependência leve e utilizadores com dependência moderada) em todas as dimensões do funcionamento familiar. Para além disso, foi possível observar associações positivas entre as dimensões do funcionamento familiar e a dependência da internet. Por fim, 
verificou-se que a comunicação na família foi a única variável do funcionamento familiar que apresentou um papel preditor na dependência da internet. Portanto, as dificuldades no funcionamento familiar, sobretudo na comunicação na família, parecem apresentar um papel fundamental no desenvolvimento da dependência da internet, sendo que se considera importante o desenvolvimento de ações preventivas, com vista a promoção do funcionamento familiar saudável, assim como do uso saudável da internet.

Palavras-chave: funcionamento familiar; dependência da internet; uso de internet; adolescentes.

\section{Abstract}

This study aimed at exploring how family functioning variables are related to internet addiction. The final sample consisted of 568 participants, aged between 14 and 18, who attend Secondary Education, in a district in the North Region of Portugal. A Sociodemographic Questionnaire, the Internet Addiction Test (IAT) and the Systemic Clinical Outcome Routine Evaluation (SCORE-15) were used. It was found that $73.1 \%$ of the participants had mild to moderate addiction, but there were no differences in internet addiction between male and female. On the other hand, differences in terms of family functioning have been verified, with male presenting slightly more difficulties in communicating in the family and more family difficulties. Differences were observed between the study groups (normal users, users with mild dependence and users with moderate dependence) in all dimensions of family functioning. In addition, it was possible to observe positive associations between the dimensions of family functioning and internet addiction. Finally, it was found that communication in the family was the only variable of family functioning that had a predictive role in internet addiction. Therefore, difficulties in family functioning, especially in communication among the members, seem to play a fundamental role in the development of internet addiction, and it is crucial to develop preventive actions, to promote healthy family functioning, as well as healthy internet use.

Keywords: family functioning; internet addiction; internet use; adolescents.

\section{Resumen}

El presente estudio tuvo como objetivo principal explorar cómo las variables del funcionamiento familiar están relacionadas con la adicción a internet. La muestra final consistió en 568 participantes, con edades comprendidas entre 14 y 18 años, que asisten a la Educación Secundaria, en un distrito en el norte de Portugal. Se utilizó cuestionario sociodemográfico, Internet Addiction Test (IAT) y Systemic Clinical Outcome Routine Evaluation (SCORE-15). Se encontró que el $73.1 \%$ de los participantes tenían una adicción leve a moderada, pero no hubo diferencias en la adicción a internet entre sexo masculino y femenino. Por otro lado, hubo diferencias en el funcionamiento familiar, ya que el género masculino tuvo un poco más de dificultades para comunicarse en la familia y más dificultades familiares. Se observaron 
diferencias entre los grupos de estudio (usuarios normales, usuarios con adicción leve y usuarios con adicción moderada) en todas las dimensiones del funcionamiento familiar. Además, fue posible observar asociaciones positivas entre las dimensiones del funcionamiento familiar y la adicción a internet. Finalmente, se descubrió que la comunicación en la familia era la única variable en el funcionamiento familiar que tenía un papel predictivo en la adicción a internet. Por lo tanto, las dificultades en el funcionamiento familiar, especialmente en la comunicación en la familia, parecen desempeñar un papel fundamental en el desarrollo de la adicción a internet, y se considera importante desarrollar acciones preventivas, con miras a promover un funcionamiento familiar saludable, así como el uso de internet saludable.

Palabras clave: funcionamiento familiar; adicción a internet; uso de internet; adolescentes.

\section{Introdução}

A utilização da internet tem vindo a aumentar exponencialmente nos últimos anos (Fernandes, Maia, \& Pontes, 2019), especialmente entre os adolescentes (Shek, Zhu, \& Dou, 2019). Em 2019, 99.5\% dos portugueses entre os 16 e 24 anos utilizava a internet (PORDATA, 2019a). Os dados divulgados pela PORDATA (2019b), indicam que o sexo masculino utiliza mais a internet do que o sexo feminino, no entanto, a diferença de utilização tornou-se menos significativa nos últimos anos, sendo que em $2019,76.8 \%$ do sexo masculino e $74.1 \%$ do sexo feminino utilizava a internet. No momento em que finalizamos o presente estudo, é provável que o número de utilizadores da internet tenha aumentado, fruto do contexto da pandemia COVID-19, que tem obrigado a uma adaptação através de aulas à distância, teletrabalho, compras online, entre outros.

A internet tornou-se um instrumento essencial para o acesso à informação, comunicação e entretenimento (Ding, Li, Zhou, Dong, \& Luo, 2017; Tsitsika et al., 2016), sendo que pode oferecer diversos benefícios (Li et al., 2020). No entanto, alguns utilizadores podem tornar-se dependentes (Jorgenson, Hsiao, \& Yen, 2016). Surge então uma nova patologia associada ao uso da internet, respetivamente, a dependência da internet (Kurniasanti, Assandi, Ismail, Nasrun, \& Wiguna, 2019).

Os adolescentes são um grupo de risco para o desenvolvimento de dependência da internet, devido à sua elevada exposição à internet sem terem desenvolvido completamente o pensamento crítico e limites comportamentais (Patrão \& Hubert, 2016), assim como por se encontrarem numa fase de intensas transformações biológicas e psicológicas (Silva \& Silva, 2017).

\section{Dependência da internet}

Até ao momento, ainda não foi alcançado nenhum consenso relativo à terminologia do fenómeno de dependência da internet ( $\mathrm{Li}$ et al., 2020). Deste modo, são usados diferentes termos na literatura como, "adição à internet" (e.g., Young, 1998), "dependência da internet" 
(e.g., Terres-Trindade \& Mosmann, 2015), "uso problemático da internet" (e.g., Fernandes et al., 2019), entre outros. Estudos recentes consideram o termo "uso problemático da internet" mais adequado (e.g., Fernandes et al., 2019). Porém, o termo "adição à internet" é amplamente usado. No presente estudo, optamos pelo uso do termo "dependência da internet", pois Pontes, Patrão e Griffiths (2014b) referem que o termo "dependência" é preferível ao de "adição".

Tal como ocorre com a terminologia, a definição do fenómeno associado à dependência da internet ainda não é estática (Lopes, Patrão, \& Gouveia, 2018). Deste modo, a dependência da internet apresenta-se como uma construção complexa (Mihajlov \& Vejmelka, 2017), que tem sido referida como um vício no espectro das dependências não-químicas do comportamento humano (Lopes et al., 2018). Segundo Young (2009), o conceito de dependência da internet foi proposto como explicação para o uso incontrolável e prejudicial da internet. Apresenta-se como uma condição onde o funcionamento do indivíduo é afetado, surgindo problemas cognitivos, psicológicos ou físicos (Karaer \& Akdemir, 2019). Os adolescentes dependentes apresentam relações familiares problemáticas, perdem relações com os amigos, deixam de realizar atividades no exterior, só se interessam por estar online, assim como apresentam alterações de comportamento, sono, alimentação e humor (Patrão, 2016). A dependência da internet ainda não foi classificada em nenhum manual de diagnóstico (Terres-Trindade \& Mosmann, 2016), sendo que até ao momento, apenas foi incluída a Perturbação de Jogos da Internet, no Manual de Diagnóstico e Estatística das Perturbações Mentais (DSM-5), com sugestão para estudos futuros (Fernandes et al., 2019). Segundo Young (1996; 2009; 2011), uma forma de diagnosticar a dependência da internet é de compará-la aos critérios diagnósticos de outras dependências estabelecidas. No entanto, o diagnóstico torna-se mais complexo, pois a internet apresenta diversas vantagens (Cruz, Scatena, Andrade, \& Micheli, 2018). Para além disso, os sinais de dependência podem ser facilmente disfarçados devido a sua utilidade (Young, 2011), assim como, podem ser interpretados como uma mudança normativa na forma como as novas gerações se ocupam ou comunicam (Kardefelt-Winther, 2014; Mihajlov \& Vejmelka, 2017).

De uma forma geral, as estimativas de prevalência de dependência da internet tendem a ser mais altas nos países asiáticos, comparativamente à Europa (Chung, Sum, \& Chan, 2019; Kurniasanti et al., 2019). Para além disso, a prevalência parece ser mais alta entre adolescentes e jovens adultos (Costa \& Patrão, 2016). No entanto, não existe uma conclusão consistente sobre as diferenças entre o sexo masculino e feminino (Shek \& Yu, 2016).

\section{Funcionamento familiar}

O funcionamento familiar apresenta-se como um constructo multifacetado, que se refere à saúde e à patologia geral do ambiente familiar (Tolou-Shams et al., 2018). Pode ser visto como as tarefas e as atividades que realizam os membros da família, de acordo com a sua posição e papel desempenhados no sistema familiar (Marín, 2007). 
Os recursos familiares, comunicação na família e dificuldades familiares são aspetos inerentes ao funcionamento familiar (Vilaça, Relvas, \& Stratton, 2017). Segundo Vilaça, Silva e Relvas (2014) os recursos familiares dizem respeito aos recursos e capacidade de adaptação da família, a comunicação na família refere-se à comunicação no sistema familiar e, por fim, as dificuldades familiares remetem para a sobrecarga das dificuldades no sistema familiar.

Uma família funcional produz condições para o desenvolvimento saudável dos seus membros (Matejevic, Jovanovic, \& Ilic, 2015). Deste modo, um funcionamento familiar de qualidade em famílias com adolescentes pode ser particularmente importante (Li, Bai, Zhang, \& Chen, 2018).

\section{Funcionamento familiar e dependência da internet}

À medida que a internet faz cada vez mais parte integrante da vida quotidiana, existe uma crescente preocupação face aos antecedentes que contribuem para a dependência da internet (Yao, He, Ko, \& Pang, 2014). Distintamente, o funcionamento familiar parece desempenhar um papel importante na dependência da internet, entre os adolescentes (Shi, Wang, \& Zou, 2017; Wartberg, Kammerl, et al., 2014).

Estudos realizados em países asiáticos com adolescentes, verificaram uma relação entre o funcionamento familiar e a dependência da internet (e.g., Chung et al., 2019; Habibi, Danesh, \& Mazandarani, 2015; Li, Garland, \& Howard, 2014; Li, Li, et al., 2018; Shi et al., 2017). Para além disso, observaram um papel preditor do funcionamento familiar na dependência da internet (e.g., Ko et al., 2015; Ko, Yen, Yen, Lin, \& Yang, 2007; Wu, Wong, et al., 2016; Yen, Yen, Chen, Chen, \& Ko, 2007; Yu \& Shek, 2013). Por outro lado, outros estudos não verificaram efeitos do funcionamento familiar sobre a dependência da internet (e.g., Shek \& Yu, 2016). Relativamente aos países europeus, a importância do papel do funcionamento familiar na dependência da internet nos adolescentes, também foi verificada (e.g., Pellerone, Ramaci, \& Heshmati, 2019; Wartberg, Kammerl, et al., 2014; Wartberg, Kriston, Bröning, Kegel, \& Thomasius, 2017). Para além disso, também se observou que os adolescentes com dependência da internet têm maior probabilidade de apresentar um funcionamento familiar menos saudável (e.g., Wartberg, Kriston, Kammerl, Petersen, \& Thomasius, 2014). Outras investigações realizadas noutros países, como na Austrália (e.g., Anderson, Steen, \& Stavropoulos, 2017), reconheceram um efeito preditor do baixo funcionamento familiar na dependência da internet. Por outro lado, no Peru, não foi verificada uma relação entre funcionamento familiar e dependência da internet (e.g., Rengifo, Ferrúa, \& Quiro, 2015).

Em Portugal, pelo conhecimento dos autores, os estudos que abordam o funcionamento familiar e a dependência da internet entre os adolescentes são escassos, sendo que as relações entre estas variáveis ainda não foram bem exploradas. As investigações realizadas neste contexto, verificaram que um maior número de situações problemáticas relacionadas com o uso das tecnologias de informação e comunicação (TIC) foram associadas a um pior funcionamento familiar (e.g., Carvalho, Francisco, \& Relvas, 2017; 2019). Para além disso, 
observaram que um nível maior de dependência da internet estava associado a um pior funcionamento familiar (e.g., Costa, Patrão, \& Machado, 2018).

A dependência da internet é considerada um problema de saúde mental sério entre os adolescentes (Ko et al., 2015) e pode agravar-se no Ensino Secundário (Nakayama, Mihara, \& Higuchi, 2017). Portanto, torna-se primordial a identificação dos fatores de risco e de proteção, associados ao fenómeno, de forma a desenvolver programas de prevenção e intervenção eficazes (Chung et al., 2019; Liu, Lin, Zhou, \& Zhang, 2019).

Deste modo, o presente estudo visa: (a) verificar a prevalência da dependência da internet na amostra; (b) explorar as diferenças na dependência da internet e nas dimensões do funcionamento familiar em função de variáveis sociodemográficas como o sexo dos participantes; (c) analisar as diferenças nas dimensões do funcionamento familiar em função do nível de dependência da internet; (d) explorar a associação entre as dimensões do funcionamento familiar e a dependência da internet; (e) e analisar o efeito preditor das dimensões do funcionamento familiar na dependência da internet.

\section{Metodologia}

O presente estudo é de caráter correlacional e quantitativo, sendo apoiado em dados de natureza numérica, recolhidos através de instrumentos de autorrelato. Também apresenta um caráter transversal, visto que os dados foram recolhidos num único momento.

\section{Participantes}

Trata-se de uma amostra de conveniência pelo que não cumpre os requisitos de amostra aleatória. A amostra final consistiu em 568 participantes, com idades compreendidas entre os 14 e 18 anos $(M=16.22 ; D P=1.03)$, dos quais 310 do sexo feminino $(54.6 \%)$ e 258 do sexo masculino (45.4\%). Todos os participantes são estudantes em Escolas de Ensino Secundário, num distrito da região Norte de Portugal, sendo que $25.9 \%$ frequenta o 10. . ano, $39.8 \%$ o 11. ano, e $34.3 \%$ o 12. ano. No que toca a idade de início de utilização da internet, esta variou entre os 3 e 15 anos $(M=8.65 ; D P=2.22)$. Por sua vez, o tempo de ligação à internet durante o dia variou entre 1 a 24 horas $(M=5.40 ; D P=3.53)$. A maior parte dos participantes $(96.8 \%)$ considera a internet importante e $40.3 \%$ julga-se dependente da mesma.

\section{Instrumentos}

No presente estudo, foi usado um Questionário Sociodemográfico, com vista a caracterização dos participantes (através de variáveis como: sexo, idade e ano de escolaridade) e da sua utilização da internet (através de variáveis como: tempo de utilização e importância da mesma).

O Internet Addiction Test (IAT), desenvolvido por Young (1998), adaptado e validado para a população portuguesa por Pontes et al. (2014b), foi utilizado, no presente estudo, para avaliar 
a dependência da internet. Este permite classificar o comportamento de dependência a nível de comprometimento leve, moderado e grave, sendo que quanto maior a pontuação, maior será o nível de dependência (Young, 2011). Neste sentido, considera-se um utilizador normal entre 0 a 30 pontos, um utilizador com dependência leve entre 31 a 49 pontos, um utilizador com dependência moderada entre 50 a 79 pontos, e um utilizador com dependência grave entre 80 a 100 pontos (Pontes et al., 2014b; Young, 2011). O IAT apresenta-se como um questionário de autorrelato composto por 20 itens, classificados segundo uma escala de Likert de 6 pontos, que varia de 0 que corresponde a "não aplicável", e 1 que corresponde a "nunca", a 5 que corresponde a "sempre". O participante deve responder tendo em conta apenas a utilização para fins recreativos e de entretenimento (Young, 2011). No presente estudo, o instrumento apresentou propriedades psicométricas boas, sendo o coeficiente alfa de Cronbach de .85 .

Para avaliar o funcionamento familiar, recorreu-se ao Systemic Clinical Outcome Routine Evaluation (SCORE-15), desenvolvido por Stratton, Bland, Janes e Lask (2010), adaptado e validado para a população portuguesa por Vilaça et al. (2014). Este permite uma avaliação de diferentes aspetos, englobados em três dimensões do funcionamento familiar, designadamente, os recursos familiares, a comunicação na família e as dificuldades familiares (Vilaça et al., 2017; Vilaça et al., 2014). É um questionário de autorresposta (Vilaça et al., 2014), composto por 15 itens respondidos em função de uma escala de Likert de 5 pontos, que varia de 1 que corresponde a "descreve-nos: muito bem" a 5 que corresponde a "descreve-nos: muito mal". No presente estudo, tal como no estudo de adaptação e validação para a população portuguesa, procedeu-se a inversão dos itens negativos $(2,4,5,7,8,9,11,12,13$ e 14), e desta forma, as pontuações mais elevadas correspondem a maiores dificuldades na família, isto é, pior funcionamento familiar. No presente estudo, o instrumento apresentou propriedades psicométricas satisfatórias, sendo o coeficiente alfa de Cronbach de .84 para a escala total, .79 para a dimensão recursos familiares, .61 para a dimensão comunicação na família e .67 para a dimensão dificuldades familiares.

\section{Procedimento}

Em primeiro lugar, foi elaborado um pedido de autorização à Comissão de Ética da Universidade de Trás-os-Montes e Alto Douro (CE-UTAD), que segue os princípios da Declaração de Helsínquia (Princípios ICH-GCP), e as Diretrizes da Schedule Y / ICMR, da Convenção de Oviedo, bem como as normas éticas da APA. Após o parecer emitido, foi realizado um pedido de autorização à Direção-Geral da Educação (DGE), através da plataforma Monitorização de Inquéritos em Meio Escolar (MIME). Após aprovação, foi elaborado um pedido de autorização para a recolha de dados às Direções das Escolas Secundárias. De seguida, foram contactados os Diretores de Turma, solicitando a sua colaboração para a entrega dos Consentimentos Informados. Posteriormente, foi combinado, com os mesmos, um horário para aplicação do protocolo aos alunos. Esta foi desenvolvida em contexto de sala de aula, com a presença da investigadora, tendo a duração de cerca de 25 minutos. 


\section{Estratégias de análise de dados}

O tratamento dos dados foi realizado com recurso ao programa Statistical Package for the Social Sciences (IBM SPSS Statistics), versão 25.0 e 26.0. Numa fase inicial, após a construção da base de dados, procedeu-se a sua limpeza, através da identificação de missings e outliers, prejudiciais à análise estatística e prosseguimento do estudo. No que respeita aos missings, no caso de um participante apresentar mais de $10 \%$ das respostas ausentes, foi retirado da amostra. Por outro lado, o reconhecimento dos outliers foi realizada com base em análises univariadas, recorrendo à determinação do Z-score, mas também em análises multivariadas, recorrendo à distância de Mahalanobis. Assim, excluíram-se 155 participantes da amostra. A normalidade foi testada com recurso ao processo de inferência estatística da distribuição normal, analisando os valores de skeweness (assimetria) e kurtosis (curtose). Para além disso, foram realizadas análises psicométricas, com vista a verificação da confiabilidade dos instrumentos, através do coeficiente alfa de Cronbach. Segundo Pallant (2005), o valor do coeficiente de alfa de Cronbach, idealmente, deve estar acima de .70. No entanto, Balbinotti e Barbosa (2008) indicam que valores superiores a .60 podem ser interpretados como satisfatórios. No presente estudo foram considerados valores superiores a .60 como sendo satisfatórios.

Relativamente à análise estatística, recorreu-se ao uso de testes paramétricos, visto que são robustos à violação do pressuposto da normalidade (Marôco, 2018). Deste modo, foram realizadas análises diferenciais, através de análises de variância multivariada (MANOVA's), onde foi identificado o valor eta parcial ao quadrado, que indica um efeito pequeno quando apresenta valores de .01, um efeito moderado quando apresenta valores de .06, ou um efeito grande quando apresenta valores de .14 (Cohen, 1998). Mas, também se recorreu ao teste-t para amostras independentes. Para além disso, procedeu-se a realização de correlações de Pearson, identificando a magnitude do efeito, onde correlações com valores entre .10 e .29 são consideradas baixas, com valores entre .30 e .49 são médias, e com valores entre .50 , e 1 são elevadas (Cohen, 1988). Finalmente, procedeu-se à realização de uma regressão múltipla standard.

\section{Resultados}

Os resultados abaixo referem-se à amostra em estudo, designadamente, 568 participantes (e.g., adolescentes que frequentam o Ensino Secundário, num distrito da região Norte de Portugal), cuja descrição já foi mencionada no tópico participantes.

\section{Prevalência de dependência da internet na amostra}

No presente estudo, segundo os critérios de Young (2011), foram encontrados 3 níveis de dependência da internet, respetivamente, utilizadores normais ( $n=153 ; 26.9 \%$ ), utilizadores com dependência leve $(n=318 ; 56 \%)$ e utilizadores com dependência moderada $(n=97 ; 17.1 \%)$. Assim, não foram identificados utilizadores com dependência grave. 


\section{Análise diferencial da dependência da internet em função do sexo do participante}

Foi realizado um teste- $t$ para amostras independentes, para explorar as diferenças entre o sexo feminino e o sexo masculino relativamente ao score de dependência da internet. A observação dos resultados permitiu verificar que não existem diferenças, estatisticamente significativas, entre os adolescentes do sexo feminino $(M=37.41 ; D P=11.10)$ e os adolescentes do sexo masculino $(M=37.48 ; D P=12.33)$, relativamente aos scores de dependência da internet $[t(563)=-.072 ; p=.943(2$-tailed $)]$.

\section{Análise diferencial das dimensões do funcionamento familiar em função do sexo do participante}

Procedeu-se a uma análise de variância multivariada (MANOVA) de forma a analisar as diferenças entre o sexo feminino e o sexo masculino nas dimensões do funcionamento familiar.

Encontraram-se diferenças, estatisticamente significativas, entre o sexo masculino e o sexo feminino $\left[F(3,564)=4.248 ; p=.006 ; \eta p^{2}=.022\right]$. Analisando os resultados para as variáveis dependentes, separadamente, verificam-se diferenças, estatisticamente significativas, na comunicação na família $[F(1,566)=9.963 ; p=.002]$, com magnitude do efeito pequena $\left(\eta p^{2}=\right.$ .017), assim como nas dificuldades familiares $[F(1,566)=7.524 ; p=.006]$, com magnitude do efeito pequena $\left(\eta p^{2}=.013\right)$. A análise das médias marginais estimadas indicou que os adolescentes do sexo masculino revelam, ligeiramente, mais dificuldades na comunicação na família, apresentando uma média superior $(M=2.07 ; D P=.66)$ aos adolescentes do sexo feminino $(M=1.90 ; D P=.60)$. Para além disso, o sexo masculino também apresenta, ligeiramente, mais dificuldades familiares, revelando uma média superior $(M=1.94 ; D P=.70)$ ao sexo feminino $(M=1.79 ; D P=.63)$ (Tabela 1$)$.

Tabela 1. Análise Diferencial das Dimensões do Funcionamento Familiar em Função do Sexo do Participante.

\begin{tabular}{ccccc}
\hline SCORE-15 & Sexo & $\mathbf{M} \pm \mathbf{D P}$ & $\boldsymbol{I C ~ 9 5 \%}$ & Direção das diferenças significativas \\
\hline \multirow{2}{*}{ Recursos Familiares } & 1- Masculino & $1.66 \pm .58$ & {$[1.59,1.74]$} & n. s. \\
& 2- Feminino & $1.64 \pm .61$ & {$[1.57,1.70]$} & \multirow{2}{*}{$1>2$} \\
\hline Comunicação na & 1- Masculino & $2.07 \pm .66$ & {$[1.99,2.15]$} & \\
Família & 2- Feminino & $1.90 \pm .60$ & {$[1.83,1.97]$} & $1>2$ \\
\hline Dificuldades & 1- Masculino & $1.94 \pm .70$ & {$[1.86,2.03]$} & \\
Familiares & 2- Feminino & $1.79 \pm .63$ & {$[1.72,1.87]$} & \\
\hline
\end{tabular}

Nota: SCORE-15 = Systemic Clinical Outcome Routine Evaluation; M = Média; DP = Desvio-padrão; IC 95\% = Intervalo de Confiança a $95 \%$. 


\section{Análise diferencial das dimensões do funcionamento familiar em função do nível de dependência da internet}

Realizou-se uma análise de variância multivariada (MANOVA), de forma a explorar as diferenças relativas às dimensões do funcionamento familiar em função do nível de dependência da internet.

De acordo com os resultados observados (Tabela 2), verificam-se diferenças, estatisticamente significativas, entre os grupos em estudo (utilizadores normais, utilizadores com dependência leve e utilizadores com dependência moderada) nas dimensões do funcionamento familiar $\left[F(6,1126)=6.245 ; p=.000 ; \eta p^{2}=.032\right]$. Considerando os resultados para as variáveis dependentes, individualmente, verificaram-se diferenças significativas entre os grupos em todas as variáveis dependentes, especificamente, nos recursos familiares $[F(2,565)=9.800$; $\left.p=.000 ; \eta p^{2}=.034\right]$, na comunicação na família $\left[F(2,565)=14.432 ; p=.000 ; \eta p^{2}=.049\right]$ e nas dificuldades familiares $\left[F(2,565)=12.689 ; p=.000 ; \eta p^{2}=.043\right]$.

Tabela 2. Análise Diferencial das Dimensões do Funcionamento Familiar em Função do Nível de Dependência da Internet.

\begin{tabular}{|c|c|c|c|c|}
\hline SCORE-15 & Grupos & $M \pm D P$ & IC 95\% & $\begin{array}{c}\text { Direção das diferenças } \\
\text { significativas }\end{array}$ \\
\hline \multirow{3}{*}{ Recursos familiares } & 1- Utilizador Normal & $1.53 \pm .57$ & {$[1.44,1.63]$} & \multirow{3}{*}{$3>2>1$} \\
\hline & 2- Dependência Leve & $1.64 \pm .58$ & {$[1.57,1.70]$} & \\
\hline & 3- Dependência Moderada & $1.87 \pm .62$ & {$[1.75,1.98]$} & \\
\hline \multirow{3}{*}{$\begin{array}{l}\text { Comunicação na } \\
\text { família }\end{array}$} & 1- Utilizador Normal & $1.79 \pm .58$ & {$[1.69,1.89]$} & \multirow{3}{*}{$3>2>1$} \\
\hline & 2- Dependência Leve & $2.00 \pm .62$ & {$[1.93,2.07]$} & \\
\hline & 3- Dependência Moderada & $2.21 \pm .68$ & {$[2.09,2.34]$} & \\
\hline \multirow{3}{*}{$\begin{array}{l}\text { Dificuldades } \\
\text { familiares }\end{array}$} & 1- Utilizador Normal & $1.71 \pm .64$ & {$[1.61,1.81]$} & \multirow{3}{*}{$3>2>1$} \\
\hline & 2- Dependência Leve & $1.85 \pm .64$ & {$[1.78,1.92]$} & \\
\hline & 3- Dependência Moderada & $2.13 \pm .69$ & {$[2.00,2.26]$} & \\
\hline
\end{tabular}

Nota: SCORE-15 = Systemic Clinical Outcome Routine Evaluation; M = Média; DP = Desvio-padrão; IC 95\% = Intervalo de Confiança a $95 \%$.

Uma análise às médias marginais estimadas permitiu identificar um aumento médio das dificuldades nos recursos familiares, comunicação na família e dificuldades familiares no grupo de utilizadores com dependência leve em comparação ao de utilizadores normais, e no grupo de utilizadores com dependência moderada em comparação ao de utilizadores com dependência leve. Devido à presença de uma variável independente com três níveis, foram realizadas análises post-hoc, recorrendo ao teste de Scheffé. As análises indicaram diferenças, estatisticamente significativas, na comunicação na família entre todos os grupos em estudo. 
Por outro lado, verificaram-se diferenças, estatisticamente significativas, nos recursos familiares e dificuldades familiares entre os grupos de utilizadores normais e utilizadores com dependência moderada, assim como entre os grupos de utilizadores leves e utilizadores com dependência moderada, não se verificando diferenças, estatisticamente significativas, entre os grupos de utilizadores normais e utilizadores com dependência leve.

\section{Associação entre dimensões do funcionamento familiar e dependência da internet}

Desenvolveu-se uma correlação de Pearson para investigar a associação entre as dimensões do funcionamento familiar e a dependência da internet.

Encontraram-se associações, estatisticamente significativas, positivas baixas entre os recursos familiares e a dependência da internet $(r=.193 ; p<.01)$, entre a comunicação na família e a dependência da internet $(r=.260 ; p<.01)$ e entre as dificuldades familiares e a dependência da internet $(r=.239 ; p<.01)$ (Tabela 3$)$.

Para além disso, verificaram-se associações, estatisticamente significativas, positivas elevadas entre todas as dimensões do SCORE-15.

Tabela 3. Associação entre Dimensões do Funcionamento Familiar e Dependência da Internet.

\begin{tabular}{lccccc}
\hline & $\mathbf{1}$ & $\mathbf{2}$ & $\mathbf{3}$ & $\mathbf{4}$ & $\mathbf{M} \pm \mathbf{D P}$ \\
\hline 1.IAT (Dependência da internet) & - & & & $37.45 \pm 11.78$ \\
\hline SCORE-15 & & & & & \\
\hline 2. Recursos Familiares & $.193^{* *}$ & - & & & $1.65 \pm .59$ \\
\hline 3. Comunicação na Família & $.260^{* *}$ & $.504^{* *}$ & - & & $1.98 \pm .63$ \\
\hline 4. Dificuldades Familiares & $.239^{* *}$ & $.511^{* *}$ & $.674^{* *}$ & - & $1.86 \pm .66$ \\
\hline
\end{tabular}

Nota: $\mathrm{M}=$ Média; DP = Desvio-padrão; ${ }^{*} \mathrm{p}<.01$.

\section{Análises preditivas: Efeito preditor das dimensões do funcionamento familiar na dependência da internet}

Realizou-se uma regressão múltipla standard, com vista a explorar a capacidade preditiva das dimensões do funcionamento familiar na dependência da internet.

O modelo final é significativo e explica uma pequena proporção (7.8\%) da variabilidade da dependência da internet $\left[F(3,564)=15.807 ; p=.000 ; R^{2}=.078 ; R^{2}{ }_{a}=.073\right]$. A análise individual das variáveis independentes permitiu identificar apenas uma variável como preditor positivo, estatisticamente significativo, da dependência da internet, respetivamente, a comunicação na família ( $\beta=.164 ; p=.004)$. Desta forma, os recursos familiares $(\beta=.061 ; p=.213$ ) e as 
dificuldades familiares ( $\beta=.097 ; p=.089)$ não apresentaram uma contribuição estatisticamente significativa (Tabela 4 ).

Tabela 4. Papel Preditor das Dimensões do Funcionamento Familiar na Dependência da Internet.

\begin{tabular}{|c|c|c|c|c|c|c|c|}
\hline & $R^{2}$ & $R^{2} a$ & B & $S E$ & $\beta$ & $t$ & $p$ \\
\hline \multicolumn{8}{|l|}{ Dependência da Internet } \\
\hline Modelo & .078 & .073 & & & & & \\
\hline Recursos Familiares & & & 1.203 & .964 & .061 & 1.248 & .213 \\
\hline $\begin{array}{l}\text { Comunicação na } \\
\text { Família }\end{array}$ & & & 3.056 & 1.052 & .164 & 2.906 & .004 \\
\hline Dificuldades Familiares & & & 1.717 & 1.008 & .097 & 1.703 & .089 \\
\hline
\end{tabular}

Nota: nível de significância de *p<.05.

\section{Discussão}

O presente estudo teve como objetivo principal explorar de que forma as variáveis do funcionamento familiar se relacionam com a dependência da internet, assim como verificar o efeito preditor das mesmas sobre a referida dependência. De forma mais específica, pretendeu verificar a prevalência de dependência da internet, assim como explorar as diferenças na dependência da internet e no funcionamento familiar em função do sexo dos participantes, e ainda analisar as diferenças nas variáveis do funcionamento familiar em função do nível de dependência.

Relativamente à prevalência de dependência da internet, de acordo com os critérios mais recentes (Young, 2011), os nossos resultados indicaram a ausência de utilizadores com dependência grave. Porém, a taxa de participantes que pertencem aos grupos de utilizadores com dependência leve e moderada $(73.1 \% ; n=415)$ parece muito elevada. Os resultados são semelhantes aos obtidos por Ferreira, Relva e Fernandes (2018), realizado com uma amostra portuguesa com idades compreendidas entre os 18 e 35 anos (77.6\%; $n=303)$, e por Pontes et al. (2014b) realizado com adolescentes e jovens adultos portugueses $(60 \% ; n=303)$. Por outro lado, outros estudos, realizados com adolescentes portugueses, verificaram a prevalência de $13 \%(n=17)$ (e.g., Pontes, Griffiths, \& Patrão, 2014a), $2 \%(n=14)$ (e.g., Ferreira et al., 2017), e $1.1 \%(n=2)$ (e.g., Carochinho \& Lopes, 2016) de utilizadores com dependência grave, mas também, de $16.5 \%$ (e.g., Martins et al., 2019) de utilizadores com dependência moderada a grave. Deste modo, as estimativas de prevalência de dependência da internet, entre os adolescentes portugueses, analisadas através dos diferentes estudos, remetem para a transformação do fenómeno numa problemática que não deve ser ignorada. 
No que concerne às diferenças entre o sexo masculino e o sexo feminino relativamente ao score de dependência da internet, os resultados são mistos. Os nossos resultados são consistentes aos obtidos por vários autores (e.g., Dufour et al., 2017; Rueda, Chávez, \& Estrella, 2017; Tran et al., 2017) em que foi utilizado o IAT, ou outros instrumentos, nomeadamente, o Young Diagnostic Questionnaire (YDQ) (e.g., Wartberg et al., 2017) e o Index of Problematic Online Experiences (I-POE) (e.g., Mitchell, Jones, \& Wells, 2013), sendo que não foram encontradas diferenças entre o sexo masculino e o sexo feminino. Porém, outros estudos encontraram diferenças, sendo que alguns indicaram maior prevalência de dependência da internet no sexo masculino, utilizando o IAT (e.g., Bhumika \& Singh, 2018; Carochinho \& Lopes, 2016; Chen et al., 2016; Dogan, Bozgeyikli, \& Bozdas, 2015; Lee et al., 2017; Lu et al., 2018; Martins et al., 2019; Pontes et al., 2014b; Stavropoulos et al., 2018; Wu, Zhang, et al., 2016; Xin et al., 2018; Yu, Kim, \& Hay, 2013), ou instrumentos diferentes, como o Chen Internet Addiction Scale (CIAS) (e.g., Chang et al., 2015; Chou \& Lee, 2017; Li, Dang, Zhang, Zhang, \& Guo, 2014), o Young's 10item Internet Addiction Test (e.g., Shek \& Yu, 2016; Shek, Zhu, \& Ma, 2018; Yu \& Shek, 2013), o Internet Addiction Diagnostic Questionnaire (IADQ) (e.g., Li, Li, et al., 2018; Shi et al., 2017), o YDQ (e.g., Durkee et al., 2012; Durkee et al., 2016), o Problematic Internet Use Questionnaire (PIUQ) (e.g., El Asam, Samara, \& Terry, 2019), ou ainda, o Adolescent Pathological Internet Use Scale (APIUS) (e.g., Liu, Fang, Zhou, Zhang, \& Deng, 2013). Por outro lado, investigações conduzidas com o mesmo objetivo, identificaram maior prevalência de dependência da internet no sexo feminino, utilizando o IAT (e.g., Karaer \& Akdemir, 2019), uma medida semelhante ao IAT (e.g., Casaló \& Escario, 2019), o Shorter PROMIS Questionnaire (SPQ) (Ballarotto, Volpi, Marzilli, \& Tambelli, 2018), o Orman's Internet Addiction Survey (e.g., Afrin, Islam, Rabbiand, \& Hossain, 2017) ou ainda, a Escala de Screening de Uso Problemático de Internet (e.g., Boubeta, Ferreiro, Salgado, \& Couto, 2015). Deste modo, apesar do crescimento da investigação, não existe uma conclusão consistente sobre as diferenças na prevalência entre o sexo masculino e feminino (Shek \& Yu, 2016). A falta de coerência nos resultados pode dever-se ao uso de diferentes métodos de avaliação, assim como a diferenças culturais (Casaló \& Escario, 2019). $O$ estudo de Kuss e Griffiths (2015), verificou que o sexo parece ser um fator de risco potencial para a dependência da internet, sendo que o sexo masculino apresenta a problemática de forma mais frequente. No entanto, a dependência da internet também parece problemática para adolescentes do sexo feminino que tendem a parecer funcionais, mas que, ainda assim, sofrem de uma dependência mais oculta, sendo esta subnotificada (Kuss \& Griffiths, 2015).

As características da própria fase da adolescência, muitas vezes, expressas através de comportamentos e desejos ambivalentes, definem a forma como os adolescentes vivenciam e descrevem as suas experiências familiares (Wagner, Falcke, Silveira, \& Mosmann, 2002). A adolescência pode ser vivida como um período difícil tanto para os adolescentes como para os seus pais (Papalia \& Feldman, 2013), sendo que existe um afastamento do grupo familiar e abertura para o mundo exterior (Castrillón \& Cala, 2019). No presente estudo, verificou-se diferenças entre o sexo masculino e feminino a nível de funcionamento familiar, sendo que o sexo masculino apresentou, ligeiramente, mais dificuldades na comunicação na família e 
dificuldades familiares, comparativamente ao sexo feminino. Estes resultados são consistentes com os resultados obtidos por Liu, Fang, Deng e Zhang (2012), que verificaram que o sexo feminino apresentou uma perceção mais positiva da comunicação entre pais e filhos. Assim, é possível que o sexo feminino tenha tendência a apresentar maior consciencialização das interações entre pais e adolescentes (Liu et al., 2013). No entanto, outros estudos não encontraram diferenças entre o sexo masculino e feminino a nível do funcionamento familiar (e.g., Li, Li, et al., 2018; Liu, Yang, Hu, Zhang, \& Nie, 2020; Vilaça et al., 2017).

Uma família, pelas suas características pode permitir que o adolescente fique cada vez mais tempo online (Patrão, Reis, Gonçalves, et al., 2016). Os nossos resultados indicam diferenças entre os grupos em estudo em todas as dimensões do funcionamento familiar, sendo que se verificaram diferenças na comunicação na família entre todos os grupos e, por outro lado, verificaram-se diferenças nos recursos familiares e dificuldades familiares entre os grupos de utilizadores normais e utilizadores com dependência moderada, assim como entre os grupos de utilizadores leves e utilizadores com dependência moderada, não havendo diferenças entre os grupos de utilizadores normais e utilizadores com dependência leve. O estudo de Park, Kim e Cho (2008) utilizando pontos de corte diferentes, observou diferenças na comunicação familiar entre o grupo "não dependentes da internet" e o grupo de "possíveis dependentes da internet", e entre o grupo "não dependentes da internet" e "dependentes da internet", mas não houve diferenças entre o grupo de "dependentes da internet" e o grupo de "possíveis dependentes da internet".

Os nossos resultados contrariam investigações anteriores que não verificaram uma relação entre dependência da internet e o funcionamento familiar (e.g., Rengifo et al., 2015), pois foi possível observar associações positivas entre as dimensões do funcionamento familiar e a dependência da internet, sendo que se espera que à medida que aumentam as dificuldades no funcionamento familiar, especificamente, nos recursos familiares, comunicação na família e dificuldades familiares, aumenta a probabilidade de desenvolvimento de dependência da internet. Os resultados obtidos são consistentes com estudos anteriores (e.g. Chung et al., 2019; Habibi et al., 2015; Li et al., 2014; Li, Li, et al., 2018; Shi et al., 2017; Wartberg et al., 2017; Wartberg, Kammerl, et al., 2014) que indicam que um pior funcionamento familiar está associado à dependência da internet entre os adolescentes, ou por outras palavras, que os adolescentes que apresentam um melhor funcionamento familiar são menos propensos ao desenvolvimento de dependência da internet. Portanto, o funcionamento familiar parece exercer um papel fundamental no desenvolvimento da dependência da internet entre os adolescentes (Shi et al., 2017; Wartberg, Kammerl, et al., 2014). Assim, é possível que a internet proporcione um anonimato onde as dificuldades da vida real, como pior funcionamento familiar, podem ser disfarçadas e combatidas (Costa \& Patrão, 2016; Xiuqin et al., 2010).

Considerando as variáveis de forma específica, nomeadamente, a comunicação na família, os nossos resultados são consistentes com outros estudos (e.g., Liu et al., 2012; Liu et al., 2019; 
Rueda et al., 2017; Xu et al., 2014) que indicam que níveis mais baixos de comunicação na família estão associados a uma maior probabilidade de desenvolver dependência da internet, ou por outras palavras, que uma boa comunicação entre pais e filhos leva a uma menor probabilidade de desenvolver dependência da internet. Deste modo, é possível que os adolescentes, face a um funcionamento familiar pobre, possam procurar afeto e relacionamentos no mundo virtual, sendo que através da internet, podem estabelecer diversas relações virtuais, assim como adquirir uma sensação temporária de afeto, de inclusão e de pertença (Costa \& Patrão, 2016). Por outro lado, em outras investigações (Bhumika \& Singh, 2018; Habibi et al., 2015) não foi verificada uma relação significativa entre comunicação na família e dependência da internet.

Por fim, observou-se que a comunicação familiar foi a única variável do funcionamento familiar que apresentou um papel preditor na dependência da internet. Especificamente, espera-se que à medida que aumentam as dificuldades na comunicação na família, aumenta a probabilidade de desenvolvimento de dependência da internet. Estes resultados são consistentes com estudos anteriores (e.g., Anderson et al., 2017; Ko et al., 2007; Ko et al., 2015; Pellerone et al., 2019; Wu, Wong, et al., 2016; Yen et al., 2007; Yu \& Shek, 2013) que verificaram que um pior funcionamento familiar se apresenta como preditor da dependência da internet, entre os adolescentes. E, que observaram que a comunicação na família apresenta um papel preditor na dependência da internet dos adolescentes (e.g., Chi, Hong, \& Chen, 2020; Liu et al., 2012). Deve-se ter em consideração que o estudo de Wu, Wong, et al. (2016) indica que os relacionamentos e interações familiares positivas desempenham um papel importante na prevenção da dependência da internet. Portanto, alguns aspetos do funcionamento familiar, como a comunicação, podem influenciar o desenvolvimento de comportamentos de risco, na fase da adolescência (Pellerone et al., 2019). Por outro lado, Shek e Yu (2016) não verificaram efeitos do funcionamento familiar sobre a dependência da internet. Também Pace et al. (2014) verificaram que a comunicação familiar não foi um preditor do desenvolvimento de dependência da internet.

\section{Implicações práticas, limitações e propostas para estudos futuros}

Os resultados do presente estudo, apresentam implicações relevantes para a prevenção da dependência da internet entre os adolescentes portugueses. Portanto, tendo em conta os resultados obtidos, consideramos importante o desenvolvimento de ações e programas preventivos, em contexto escolar e familiar, no sentido de promover o funcionamento familiar saudável, e sobretudo, a comunicação na família, como forma de prevenir o desenvolvimento da dependência da internet. Para além disso, o desenvolvimento de ações para informar e alertar os adolescentes, pais, professores e população em geral sobre a problemática, promovendo o uso saudável da internet, também se torna fundamental.

O presente estudo apresenta limitações, que podem surgir como indicações e sugestões para estudos futuros. Em primeiro lugar, ainda não existe consenso quanto à definição e classificação 
para a dependência da internet (Patrão, Reis, Madeira, et al., 2016). Para além disso, os pontos de corte proposto pelo autor original da escala usada no presente estudo, são apenas especulativos, pois não foram realizados estudos empíricos ou clínicos para comprová-los (Pontes et al., 2014b). Outra limitação refere-se ao uso de questionários de autorrelato, considerando apenas a perceção dos adolescentes. Por fim, devido ao tamanho da amostra não ser representativo da população portuguesa, os resultados não podem ser generalizados.

Deste modo, em estudos futuros poderá ser interessante utilizar uma amostra mais representativa, através da inclusão de um maior número de participantes, provenientes de diversas regiões de Portugal. Também se pode tornar interessante incluir a perspetiva dos pais, de forma a adquirir uma compreensão mais rica, precisa e completa dos resultados. Para além disso, a investigação deve apresentar como prioridade o desenvolvimento de uma definição abrangente de dependência da internet, possibilitando o desenvolvimento de um instrumento psicometricamente sólido para a sua avaliação (Shek, Yu, \& Sun, 2016).

\section{Referências}

Afrin, D., Islam, M.-U., Rabbiand, F., \& Hossain, A. (2017). The school-level factors associated with internet addiction among adolescents: Across-sectional study in Bangladesh. Journal of Addiction and Dependence, 3(2), 170-174. https://doi.org/10.15436/2471061X.17.1686

Anderson, E. L., Steen, E., \& Stavropoulos, V. (2017). Internet use and problematic internet use: A systematic review of longitudinal research trends in adolescence and emergent adulthood. International Journal of Adolescence and Youth, 22(4), 430-454. https://doi.org/10.1080/02673843.2016.1227716

Balbinotti, M. A. A., \& Barbosa, M. L. L. (2008). Análise da consistência interna e fatorial confirmatório do IMPRAFE-126 com praticantes de atividades físicas gaúchos. PsicoUSF, 13(1), 1-12. https://doi.org/10.1590/S1413-82712008000100002

Ballarotto, G., Volpi, B., Marzilli, E., \& Tambelli, R. (2018). Adolescent internet abuse: A study on the role of attachment to parents and peers in a large community sample. BioMed Research Internacional, 2018(14), 1-10. https://doi.org/10.1155/2018/5769250

Bhumika, A., \& Singh, S. (2018). Internet addiction, family communication pattern and perceived social support among adolescents. Periodic Research, 7(1), 229-232.

Boubeta, A. R., Ferreiro, S. G., Salgado, P. G., \& Couto, C. B. (2015). Variables asociadas al uso problemático de internet entre adolescentes. Health and Addictions, 15(1), 25-38. https://doi.org/10.21134/haaj.v15i1.223

Carochinho, J.-A., \& Lopes, M. I. (2016). A dependência à internet nos jovens de uma escola de cariz militar. Revista de Psicologia da Criança e do Adolescente, 7(1-2), 489-507.

Carvalho, J., Francisco, R., \& Relvas, A. P. (2017). ICTs and family functioning: A study on Portuguese families with adolescents and emerging adults. Contemporary Family Therapy, 39, 281-288. https://doi.org/10.1007/s10591-017-9436-8 
Carvalho, J., Francisco, R., \& Relvas, A. P. (2019). e-Famílias: O impacto das TIC na vida contemporânea de famílias com crianças. In R. Brito, \& P. Dias, Crianças, famílias $e$ tecnologias. Que desafios? Que caminhos? (pp. 184-197). Lisboa: CIED (Centro Interdisciplinar de Estudos Educacionais).

Casaló, L. V., \& Escario, J.-J. (2019). Predictors of excessive internet use among adolescents in Spain: The relevance of the relationship between parents and their children. Computers in Human Behavior, 92, 344-351. https://doi.org/10.1016/j.chb.2018.11.042

Castrillón, J. J. C., \& Cala, M. L. P. (2019). Funcionalidad familiar y tendencias adictivas a internet y a sustancias psicoativas en estudantes universitarios. Psicología desde el Caribe, 36(2), 177-206.

Chang, F.-C., Chiu, C.-H., Miao, N.-F., Chen, P.-H., Lee, C.-M., Chiang, J.-T., \& Pan, Y.-C. (2015). The relationship between parental mediation and internet addiction among adolescents, and the association with cyberbullying and depression. Comprehensive Psychiatry, 57, 2128. https://doi.org/10.1016/j.comppsych.2014.11.013

Chen, Y., Kang, Y., Gong, W., He, L., Jin, Y., Zhu, X., \& Yao, Y. (2016). Investigation on internet addiction disorder in adolescents in Anhui, people's Republic of China. Neuropsychiatric Disease and Treatment, 2016(12), 2233-2236. https://doi.org/10.2147/NDT.S110156

Chi, X., Hong, X., \& Chen, X. (2020). Profiles and sociodemographic correlates of internet addiction in early adolescents in southern China. Addictive Behavior, 106, 1-7. https://doi.org/10.1016/i.addbeh.2020.106385

Chou, C., \& Lee, Y.-H. (2017). The moderating effects of internet parenting styles on the relationship between internet parenting behavior, internet expectancy, and internet addiction tendency. The Asia-Pacific Education Researcher, 26, 137-146. https://doi.org/10.1007/s40299-017-0334-5

Chung, T. W. H., Sum, S. M. Y., \& Chan, M. W. L. (2019). Adolescent internet addiction in Hong Kong: Prevalence, psychosocial correlates, and prevention. Journal of Adolescent Health, 64(6), S34-S43. https://doi.org/10.1016/i.jadohealth.2018.12.016

Cohen, J. (1988). Statistical power analysis for the behavioral sciences (2 ed.). Hillsdale, NJ: Lawrence Erlbaum Associates, Publishers.

Costa, R. M., \& Patrão, I. (2016). As relações amorosas e a internet: Dentro e fora da rede. In I. Patrão, \& D. Sampaio, Dependências online: O poder das tecnologias (pp. 117-132). Lisboa: PACTOR.

Costa, R. M., Patrão, I., \& Machado, M. (2018). Problematic internet use and feelings of loneliness. International Journal of Psychiatry in Clinical Practice, 23(2), 160-162. https://doi.org/10.1080/13651501.2018.1539180

Cruz, F. A. D., Scatena, A., Andrade, A. L. M., \& Micheli, D. (2018). Evaluation of internet addiction and the quality of life of Brazilian adolescents from public and private schools. Estudos de Psicologia (Campinas), 35(2), 193-204. https://doi.org/10.1590/1982$\underline{02752018000200008}$ 
Ding, Q., Li, D., Zhou, Y., Dong, H., \& Luo, J. (2017). Perceived parental monitoring and adolescent internet addiction: A moderated mediation model. Addictive Behaviors, 74, 4854. https://doi.org/10.1016/j.addbeh.2017.05.033

Dogan, H., Bozgeyikli, H., \& Bozdas, C. (2015). Perceived parenting styles as predictor of internet addiction in adolescence. International Journal of Research in Education and Science (IJRES), 1(2), 167-174. https://doi.org/10.21890/ijres.87952

Dufour, M., Brunelle, N., Khazaal, Y., Tremblay, J., Leclerc, D., Cousineau, M.-M., . . Berbiche, D. (2017). Gender difference in online activities that determine problematic internet use. Journal de Thérapie Comportementale et Cognitive, 27(3), 90-98. https://doi.org/10.1016/i.jtcc.2017.05.002

Durkee, T., Carli, V., Floderus, B., Wasserman, C., Sarchiapone, M., Apter, A., . . Wasserman, D. (2016). Pathological internet use and risk-behaviors among European adolescents. International Journal of Environmental Research and Public Health, 13(3), 1-17. https://doi.org/10.3390/ijerph13030294

Durkee, T., Kaess, M., Carli, V., Parzer, P., Wasserman, C., Floderus, B., . . . Wasserman, D. (2012). Prevalence of pathological internet use among adolescents in Europe: Demographic and social factors. Addiction, 107(12), 2210-2222. https://doi.org/10.1111/i.1360-0443.2012.03946.x

El Asam, A., Samara, M., \& Terry, P. (2019). Problematic internet use and mental health among British children and adolescents. Addictive Behaviors, 90, 428-436. https://doi.org/10.1016/j.addbeh.2018.09.007

Fernandes, B., Maia, B. R., \& Pontes, H. M. (2019). Adição à internet ou uso problemático da internet? Qual dos termos usar? Psicologia USP, 30(e190020), 1-8. https://doi.org/10.1590/0103-6564e190020

Ferreira, C., Ferreira, H., Vieira, M. J., Costeira, M., Branco, L., Dias, Â., \& Macedo, L. (2017). Epidemiologia do uso de internet numa população adolescente e sua relação com hábitos de sono. Revista Científica da Ordem dos Médicos, 30(7-8), 524-533. https://doi.org/10.20344/amp.8205

Ferreira, L. A., Relva, I. C., \& Fernandes, O. M. (2018). Adição à internet: Relação com a sintomatologia psicopatológica. PsychTech \& Health Journal, 1(2), 24-37. https://doi.org/10.26580/PTHJ.art7.2018

Habibi, A., Danesh, P., \& Mazandarani, M. J. Z. (2015). The relationship of family function with internet addiction among girl high school students in Malard. Mediterranean Journal of Social Sciences, 6(4), 215-222. https://doi.org/10.5901/mjss.2015.v6n4s3p215

Jorgenson, A. G., Hsiao, R. C.-J., \& Yen, C.-F. (2016). Internet addiction and other behavioral addictions. Child and Adolescent Psychiatric Clinics of North America, 25(3), 509-520. https://doi.org/10.1016/i.chc.2016.03.004

Karaer, Y., \& Akdemir, D. (2019). Parenting styles, perceived social support and emotion regulation in adolescents with internet addiction. Comprehensive Psychiatry, 92, 22-27. https://doi.org/10.1016/i.comppsych.2019.03.003 
Kardefelt-Winther, D. (2014). A conceptual and methodological critique of internet addiction research: Towards a model of compensatory internet use. Computers in Human Behavior, 31, 351-354. https://doi.org/10.1016/i.chb.2013.10.059

Ko, C.-H., Wang, P.-W., Liu, T.-L., Yen, C.-F., Chen, C.-S., \& Yen, J.-Y. (2015). Bidirectional associations between family factors and internet addiction among adolescents in a prospective investigation. Psychiatry and Clinical Neurosciences, 69(4), 192-200. https://doi.org/10.1111/pcn.12204

Ko, C.-H., Yen, J.-Y., Yen, C.-F., Lin, H.-C., \& Yang, H.-J. (2007). Factors predictive for incidence and remission of internet addiction in young adolescents: A prospective study. CyberPsychology \& Behavior, 10(4), 545-551. https://doi.org/10.1089/cpb.2007.9992

Kurniasanti, K. S., Assandi, P., Ismail, R. I., Nasrun, M. W. S., \& Wiguna, T. (2019). Internet addiction: A new addiction? Medical Journal of Indonesia, 28(1), 82-91. https://doi.org/10.13181/mji.v28i1.2752

Kuss, D. J., \& Griffiths, M. D. (2015). Internet addiction in psychotherapy. United Kingdom, UK: Palgrave Macmillan. https://doi.org/10.1057/9781137465078.0001

Lee, J.-Y., Kim, S.-Y., Bae, K.-Y., Kim, J.-M., Shin, I.-S., Yoon, J.-S., \& Kim, S.-W. (2017). Prevalence and risk factors for problematic internet use among rural adolescents in Korea. AsiaPacific Psychiatry, 10(2), 1-4. https://doi.org/10.1111/appy.12310

Li, C., Dang, J., Zhang, X., Zhang, Q., \& Guo, J. (2014). Internet addiction among Chinese adolescents: The effect of parental behavior and self-control. Computers in Human Behavior, 41, 1-7. https://doi.org/10.1016/j.chb.2014.09.001

Li, J., Li, D., Jia, J., Li, X., Wang, Y., \& Li, Y. (2018). Family functioning and internet addiction among adolescent males and females: A moderated mediation analysis. Children and Youth Services Review, 91, 289-297. https://doi.org/10.1016/j.childyouth.2018.06.032

Li, L., Bai, L., Zhang, X., \& Chen, Y. (2018). Family functioning during adolescence: The roles of paternal and maternal emotion dysregulation and parent-adolescent relationships. Journal of Child and Family Studies, 27(4), 1311-1323. https://doi.org/10.1007/s10826017-0968-1

Li, W., Garland, E. L., \& Howard, M. O. (2014). Family factors in internet addiction among Chinese youth: A review of English- and Chinese-language studies. Computers in Human Behavior, 31(1), 393-411. https://doi.org/10.1016/i.chb.2013.11.004

Li, Y., Wang, Y., Ren, Z., Gao, M., Liu, Q., Qiu, C., \& Zhang, W. (2020). The influence of environmental pressure on internet use disorder in adolescents: The potential mediating role of cognitive function. Addictive Behaviors, 101(105976), 1-9. https://doi.org/10.1016/i.addbeh.2019.04.034

Liu, Q., Lin, Y., Zhou, Z., \& Zhang, W. (2019). Perceived parent-adolescent communication and pathological internet use among Chinese adolescents: A moderated mediation model. Journal of Child and Family Studies, 28(2), 1571-1580. https://doi.org/10.1007/s10826$\underline{019-01376-x}$ 
Liu, Q.-Q., Yang, X.-J., Hu, Y.-T., Zhang, C.-Y., \& Nie, Y.-G. (2020). How and when is family dysfunction associated with adolescent mobile phone addiction? Testing a moderated mediation model. Children and Youth Services Review, 111, 1-9. https://doi.org/10.1016/i.childyouth.2020.104827

Liu, Q.-X., Fang, X.-Y., Deng, L.-Y., \& Zhang, J.-T. (2012). Parent-adolescent communication, parental internet use and internet-specific norms and pathological internet use among Chinese adolescents. Computers in Human Behavior, 28(4), 1269-1275. https://doi.org/10.1016/j.chb.2012.02.010

Liu, Q.-X., Fang, X.-Y., Zhou, Z.-K., Zhang, J.-T., \& Deng, L.-Y. (2013). Perceived parent-adolescent relationship, perceived parental online behaviors and pathological internet use among adolescents: Gender-specific differences. PLOS ONE, 8(9). https://doi.org/10.1371/journal.pone.0075642

Lopes, R. S., Patrão, I., \& Gouveia, M. J. (2018). Perceção de tempo online: Revisão de literatura. Psicologia, Saúde \& Doenças, 19(1), 151-156. https://doi.org/10.15309/18psd190122

Lu, L., Xu, D.-D., Liu, H.-Z., Zhang, L., Ng, C. H., Ungvari, G. S., . . Xiang, Y.-T. (2018). Internet addiction in Tibetan and Han Chinese middle school students: Prevalence, demographics and quality of life. Psychiatry Research, 268, 131-136. https://doi.org/10.1016/i.psychres.2018.07.005

Marín, J. M. (2007). Función y disfunción familiar. FMC - Formación Médica Continuada en Atención Primaria, 14(5), 89-99. https://doi.org/10.1016/S1134-2072(07)74022-7

Marôco, J. (2018). Análise estatística com o SPSS statistics (7 ed.). Pêro Pinheiro: ReportNumber Análise e Gestão de Informação.

Martins, M. V., Formiga, A., Santos, C., Sousa, D., Resende, C., Campos, R., .. Ferreira, S. (2019). Adolescent internet addiction - role of parental control and adolescent behaviours. International Journal of Pediatrics and Adolescent Medicine. https://doi.org/10.1016/i.ijpam.2019.12.003

Matejevic, M., Jovanovic, D., \& Ilic, M. (2015). Patterns of family functioning and parenting style of adolescents with depressive reactions. Procedia-Social and Behavioral Sciences, 185, 234-239. https://doi.org/10.1016/i.sbspro.2015.03.460

Mihajlov, M., \& Vejmelka, L. (2017). Internet addiction: A review of the first twenty years. Psychiatria Danubina, 29(3), 260-272. https://doi.org/10.24869/psyd.2017.260

Mitchell, K. J., Jones, L. M., \& Wells, M. (2013). Testing the Index of Problematic Online Experiences (I-POE) with a national sample of adolescents. Journal of Adolescence, 36(6), 1153-1163. https://doi.org/10.1016/i.adolescence.2013.09.004

Nakayama, H., Mihara, S., \& Higuchi, S. (2017). Treatment and risk factors of internet use disorders. Psychiatry and Clinical Neurosciences, 71(7), 492-505. https://doi.org/10.1111/pcn.12493

Pace, U., Zappulla, C., Guzzo, G., Di Maggio, R., Laudani, C., \& Cacioppo, M. (2014). Internet addiction, temperament, and the moderator role of family emotional involvement. International Journal of Mental Health and Addiction, 12(1), 52-63. https://doi.org/10.1007/s11469-013-9468-8 
Pallant, J. (2005). SPSS survival manual: A step by step guide to data analysis using SPSS. Australia: Allen \& Unwin.

Papalia, D. E., \& Feldman, R. D. (2013). Desenvolvimento humano (12 ed.). Porto Alegre: AMGH Editora Ltda.

Park, S. K., Kim, J. Y., \& Cho, C. B. (2008). Prevalence of internet addiction and correlations with family factors among south Korean adolescents. Adolescence, 43(172), 895-909.

Patrão, I. (2016). \#GeraçãoCordão: A geração que não desliga! Lisboa: PACTOR.

Patrão, I., \& Hubert, P. (2016). Os comportamentos e as preferências online dos jovens portugueses: O jogo online e as redes sociais. In I. Patrão, \& D. Sampaio, Dependências online: O poder das tecnologias (pp. 97-116). Lisboa: PACTOR.

Patrão, I., Reis, J., Gonçalves, J., Moura, B., Abreu, C. N., \& Sampaio, D. (2016). Intervenção clínica nas dependências online. In I. Patrão, \& D. Sampaio, Dependências online: O poder das tecnologias (pp. 169-186). Lisboa: PACTOR.

Patrão, I., Reis, J., Madeira, L., Paulino, M. C. S., Barandas, R., Sampaio, D., . . Carmenates, S. (2016). Avaliação e intervenção terapêutica na utilização problemática da internet (UPI) em jovens: Revisão da literatura. Revista de Psicologia da Criança e do Adolescente, 7(12), 221-243.

Pellerone, M., Ramaci, T., \& Heshmati, R. (2019). Psychology and psychopathology of the mask. Mediterranean Journal of Clinical Psychology, 7(1), 1-20. https://doi.org/10.6092/2282$\underline{1619 / 2019.7 .2237}$

Pontes, H. M., Griffiths, M. D., \& Patrão, I. M. (2014a). Internet addiction and loneliness among children and adolescents in the education setting: An empirical pilot study. Aloma: Revista de Psicologia, Ciències de l'Educació i de l'Esport, 32(1), 91-98.

Pontes, H. M., Patrão, I. M., \& Griffiths, M. D. (2014b). Portuguese validation of the Internet Addiction Test: An empirical study. Journal of Behavioral Addictions, 3(2), 107-114. https://doi.org/10.1556/JBA.3.2014.2.4

PORDATA. (2019a). Indivíduos com 16 e mais anos que utilizam computador e internet em \% do total de indivíduos: Por grupo etário - Portugal. Retirado de: https://www.pordata.pt/Portugal/Indiv\%C3\%ADduos+com+16+e+mais+anos+que+utiliz am+computador+e+Internet+em+percentagem+do+total+de+indiv\%C3\%ADduos+por+gr upo+etário-1139

PORDATA. (2019b). Indivíduos com 16 e mais anos que utilizam computador e internet em \% do total de indivíduos: Por sexo - Portugal. Retirado de: https://www.pordata.pt/Portugal/Indiv\%C3\%ADduos+com+16+e+mais+anos+que+utiliz am+computador+e+Internet+em+percentagem+do+total+de+indiv\%C3\%ADduos+por+se $\underline{x o-1142}$

Rengifo, C. A., Ferrúa, L. M., \& Quiro, W. V. (2015). Funcionamiento familiar y adicción a internet en estudiantes de uma institución educativa pública de Lima Norte. Revista Científica de Ciencias de la Salud, 8(2), 9-17. https://doi.org/10.17162/rccs.v8i2.467 
Rueda, D. R. A., Chávez, P. C. \& Estrella, J. E. G. (2017). Prevalencia de adicción a internet y su relación con disfunción familiar en adolescentes. Revista Clínica de Medicina de Familia, 10(3), 179-186.

Shek, D. T. L., \& Yu, L. (2016). Adolescent internet addiction in Hong Kong: Prevalence, change, and correlates. Journal of Pediatric \& Adolescent Gynecology, 29(1), S22-S30. https://doi.org/10.1016/j.jpag.2015.10.005

Shek, D. T. L., Yu, L., \& Sun, R. C. F. (2016). Internet addiction. In D. W. Pfaff, \& N. D. Volkow, Neuroscience in the 21st century (pp. 3707-3750). New York: Springer. https://doi.org/10.1007/978-1-4939-3474-4 108

Shek, D. T. L., Zhu, X., \& Dou, D. (2019). Influence of family processes on internet addiction among late adolescents in Hong Kong. Frontiers in Psychiatry, 10(113), 1-17. https://doi.org/10.3389/fpsyt.2019.00113

Shek, D. T. L., Zhu, X., \& Ma, C. M. S. (2018). The influence of parental control and parent-child relational qualities on adolescent internet addiction: A 3-year longitudinal study in Hong Kong. Frontiers in Psychology, 9(642). https://doi.org/10.3389/fpsyg.2018.00642

Shi, X., Wang, J., \& Zou, H. (2017). Family functioning and internet addiction among Chinese adolescents: The mediating roles of self-esteem and loneliness. Computers in Human Behavior, 76, 201-210. https://doi.org/10.1016/i.chb.2017.07.028

Silva, T. O., \& Silva, L. T. G. (2017). Os impactos sociais, cognitivos e afetivos sobre a geração de adolescentes conectados às tecnologias digitais. Revista Psicopedagogia, 34(103), 87-97.

Stavropoulos, V., Griffiths, M. D., Burleigh, T. L., Kuss, D. J., Doh, Y. Y., \& Gomez, R. (2018). Flow on the internet: A longitudinal study of internet addiction symptoms during adolescence.

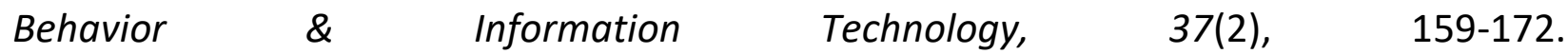
https://doi.org/10.1080/0144929X.2018.1424937

Stratton, P., Bland, J., Janes, E., \& Lask, J. (2010). Developing an indicator of family function and a practicable outcome measure for systemic family and couple therapy: The SCORE. Journal of Family Therapy, 32(3), 232-258. https://doi.org/10.1111/i.14676427.2010.00507.x

Terres-Trindade, M., \& Mosmann, C. P. (2015). Discriminant profile of young internet dependents: The role of family relationships. Paidéia, 25(62), 353-361. https://doi.org/10.1590/1982-43272562201509

Terres-Trindade, M., \& Mosmann, C. P. (2016). Conflitos familiares e práticas educativas parentais como preditores de dependência da internet. Psico-USF, 21(3), 623-633. https://doi.org/10.1590/1413-82712016210315

Tolou-Shams, M., Brogan, L., Esposito-Smythers, C., Healy, M. G., Lowery, A., Craker, L., \& Brown, L. K. (2018). The role of family functioning in parenting practices of court- involved youth. Journal of Adolescence, 165-174. https://doi.org/10.1016/i.adolescence.2017.12.016 
Tran, B. X., Huong, L. T., Hinh, N. D., Nguyen, L. H., Le, B. N., Nong, V., . . Ho, R. C. (2017). A study on the influence of internet addiction and online interpersonal influences on healthrelated quality of life in young Vietnamese. BMC Public Health, 17(138), 1-8. https://doi.org/10.1186/s12889-016-3983-z

Tsitsika, A. K., Andrie, E. K., Psaltopoulou, T., Tzavara, C. K., Sergentanis, T. N., NtanasisStathopoulos, I., . . . Tsolia, M. (2016). Association between problematic internet use, sociodemographic variables and obesity among European adolescents. The European Journal of Public Health, 26(4), 617-622. https://doi.org/10.1093/eurpub/ckw028

Vilaça, M., Relvas, A. P., \& Stratton, P. (2017). A Portuguese translation of the Systemic Clinical Outcome and Routine Evaluation (SCORE): The psychometric properties of the 15- and 28item versions. Journal of Family Therapy, 40(4), 537-556. https://doi.org/10.1111/1467$\underline{6427.12197}$

Vilaça, M., Silva, J. T., \& Relvas, A. P. (2014). Systemic Clinical Outcome Routine Evaluation (SCORE-15). In A. Relvas, \& S. Major, Instrumentos deAvaliação familiar: Funcionamento e intervenção (Vol. I) (pp. 23-41). Coimbra: Imprensa da Universidade de Coimbra. https://doi.org/10.14195/978-989-26-0839-6 1

Wagner, A., Falcke, D., Silveira, L. M. B. O., \& Mosmann, C. P. (2002). A comunicação em famílias com filhos adolescentes. Psicologia em Estudo, 7(1), 75-80. https://doi.org/10.1590/S1413-73722002000100010

Wartberg, L., Kammerl, R., Rosenkranz, M., Hirschhäuser, L., Hein, S., Schwinge, C., . . . Thomasius, R. (2014). The interdependence of family functioning and problematic internet use in a representative quota sample of adolescents. Cyberpsychology, Behavior, and Social Networking, 17(1), 14-18. https://doi.org/10.1089/cyber.2012.0494

Wartberg, L., Kriston, L., Bröning, S., Kegel, K., \& Thomasius, R. (2017). Adolescent problematic internet use: Is a parental rating suitable to estimate prevalence and identify familial correlates? Computers in Human Behavior, 67, 233-239. https://doi.org/10.1016/i.chb.2016.10.029

Wartberg, L., Kriston, L., Kammerl, R., Petersen, K.-U., \& Thomasius, R. (2014). Prevalence of pathological internet use in a representative German sample of adolescents: Results of a latent profile analysis. Psychopathology, 48(1), 25-30. https://doi.org/10.1159/000365095

Wu, C. S. T., Wong, H. T., Yu, K. F., Fok, K.W., Yeung, S. M., Lam, C. H., \& Liu, K. M. (2016). Parenting approaches, family functionality, and internet addiction among Hong Kong adolescents. BMC Pediatrics, 16(130), 1-10. https://doi.org/10.1186/s12887-016-0666-y

Wu, X.-S., Zhang, Z.-H., Zhao, F., Wang, W.-J., Li, Y.-F., Bi, L., . . Sun, Y.-H. (2016). Prevalence of internet addiction and its association with social support and other related factors among adolescents in China. Journal of Adolescence, 52, 103-111. https://doi.org/10.1016/i.adolescence.2016.07.012 
Xin, M., Xing, J., Pengfei, W., Houru, L., Mengcheng, W., \& Hong, Z. (2018). Online activities, prevalence of internet addiction and risk factors related to family and school among adolescents in China. Addictive Behaviors Reports, 7, 14-18. https://doi.org/10.1016/i.abrep.2017.10.003

Xiuqin, H., Huimin, Z., Mengchen, L., Jinan, W., Ying, Z., \& Ran, T. (2010). Mental health, personality, and parental rearing styles of adolescents with internet addiction disorder. Cyberpsychology, Behavior, and Social Networking, 13(4), 401-406. https://doi.org/10.1089/cyber.2009.0222

Xu, J., Shen, L.-X., Yan, C.-H., Hu, H., Yang, F., Wang, L., . . Shen, X.-M. (2014). Parent-adolescent interaction and risk of adolescent internet addiction: A population-based study in Shanghai. BMC Psychiatry, 14(112). https://doi.org/10.1186/1471-244X-14-112

Yao, M. Z., He, J., Ko, D. M., \& Pang, K. (2014). The influence of personality, parental behaviors, and self-esteem on internet addiction: A study of Chinese college students. CyberPsychology, Behavior, and Social Networking, 17(2), 104-110. https://doi.org/10.1089/cyber.2012.0710

Yen, J.-Y., Yen, C.-F., Chen, C.-C., Chen, S.-H., \& Ko, C.-H. (2007). Family factors of internet addiction and substance use experience in Taiwanese adolescents. CyberPsychology \& Behavior, 10(3), 323-329. https://doi.org/10.1089/cpb.2006.9948

Young, K. S. (1996). Psychology of computer use: XL. Addictive use of the internet: A case that breaks the stereotype. Psychological Reports, 79, 899-902. https://doi.org/10.2466/pr0.1996.79.3.899

Young, K. S. (1998). Internet addiction: The emergence of a new clinical disorder. Cyberpsychology \& Behavior, 1(3), 237-244. https://doi.org/10.1089/cpb.1998.1.237

Young, K. S. (2009). Internet addiction: Diagnosis and treatment considerations. Journal of Contemporary Psychotherapy, 39(4), 241-246. https://doi.org/10.1007/s10879-009-9120$\underline{\mathrm{x}}$

Young, K. S. (2011). Clinical assessment of internet-addicted clients. In K. S. Young, \& C. N. Abreu, Internet addiction: A handbook and guide to evaluation and treatment (pp. 19-34). New Jersey: John Wiley \& Sons. https://doi.org/10.1002/9781118013991.ch2

Yu, J. J., Kim, H., \& Hay, I. (2013). Understanding adolescents' problematic internet use from a social/ cognitive and addiction research framework. Computers in Human Behavior, 29(6), 2682-2689. https://doi.org/10.1016/i.chb.2013.06.045

Yu, L., \& Shek, D. T. L. (2013). Internet addiction in Hong Kong adolescents: A three-year longitudinal study. Journal of Pediatric \& Adolescent Gynecology, 26(3), S10-S17. https://doi.org/10.1016/i.jpag.2013.03.010 